\title{
A method of jellyfish detection based on high resolution multi- beam acoustic image
}

\author{
Yang Zhang, Guijuan Li*, Mingwei Zhang, and Yi Jiang \\ Science and Technology on Underwater Test and Control Laboratory, Dalian, China
}

\begin{abstract}
A method based on high resolution multi-beam acoustic image was brought forward for jellyfish detection, and a moon jellyfish detection experiment was conducted on sea. In this experiment, clear acoustic images of individual moon jellyfish were obtained, acoustic method was verified available in moon jellyfish detection. Echo characteristics of individual moon jellyfish were analysed. In the echo image of individual moon jellyfish, four evident echo highlights were found, which were due to the four stomach pouches. Three acoustic characteristic parameters were proposed and estimated to describe jellyfish acoustic characteristics, which were acoustic diameter, acoustic height, and relative target strength. In addition, dynamic characteristic of moon jellyfish was also studied during its pulse motion.
\end{abstract}

\section{Introduction}

Jellyfish blooming is considered one of the most serious ecological disasters in East Asia, which seriously affects the marine ecosystem, environmental security and marine economic development [1] Jellyfish detection and early-warning techniques are being paid more and more attention. Current methods of jellyfish monitoring include optical imaging and jellyfish hydroids monitoring [2-3]. Due to the rapid attenuation of light in the water, the monitoring scope is limited, hydroids monitoring is also difficult to detect and monitor jellyfish outbreaks in real time. As the only carrier which is capable of transmitting and carrying information underwater, the sound wave can be used to detect jellyfish. In this paper a jellyfish detection method based on high-resolution multi-beam acoustic image was proposed. And a jellyfish detection test was carried out on the sea.

\section{The jellyfish detection test}

The jellyfish detection test on sea was designed and conducted to obtain the echo data of jellyfish.

A high-resolution multi-beam sonar (referred to as sonar) was used in the test. The sonar working at 400 $\mathrm{kHz}$ frequency can launch underwater high-resolution multi-beam, with a beam resolution of $0.4^{\circ} \times 0.4^{\circ}$ (Fig.1(a)).

The test was conducted on the sea, on August 24, 2016. The experimental platform is a wooden fishing boat, which was about 15 meters in length.

The sonar was fixed about 3 meters below the sea surface, and the detecting sound beam was ejected straight forward, and the target swam in the front of the sonar beam. The jellyfish detection test schematic diagram is shown in Fig.1(b).

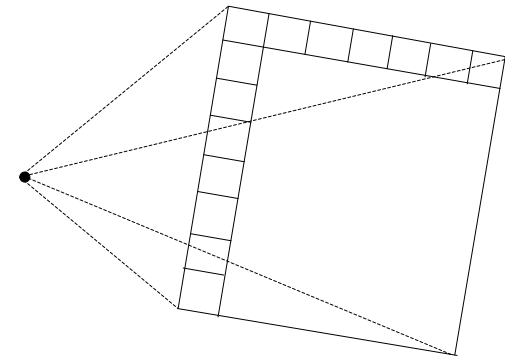

(a) Picture of the sonar beams

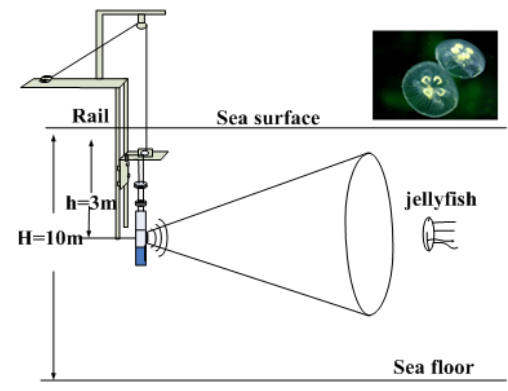

(b) The schematic diagram of jellyfish detection test

Fig. 1. The sonar beams and the overseas test schematic.

\section{Method of data analysis and proce- ssing}

Firstly, acoustic image of individual jellyfish is abstracted from the raw echo image. Secondly, three parameters are brought up to describe characteristics of moon jellyfish. Finally, the dynamical characteristics of moon jellyfish are analysed.

According to the sonar equation

$$
\begin{aligned}
\mathrm{EL} & =\mathrm{SL}-2 \mathrm{TL}+\mathrm{TS} \\
\mathrm{L} & =20 \log \mathrm{R}
\end{aligned}
$$


where EL is the combinatorial sonar parameter echo signal energy level, SL is the source level of the sonar; TL is a one-way propagation loss, which is in the spherical wave attenuation, and the absorption loss of the sea water is ignored; $\mathrm{R}$ is the target distance; $\mathrm{TS}$ is the target strength.

To describe the ability of jellyfish to reflect sound waves, the relative echo strength (RES) of jellyfish is proposed in the paper. RES is related to the source level (SL) and target strength TS according to the equation:

$$
\mathrm{RES}=\mathrm{SL}+\mathrm{TS}=\mathrm{EL}+2 \mathrm{TL}
$$

To detect jellyfish effectively, two other parameters are also proposed: the diameter (De) and height $(\mathrm{He})$ of jellyfish in the acoustic image. Figure 2 is the diameter and height of one jellyfish in optical picture and echo image. In the optical picture, the height is about half the diameter of the umbrella body. In the echo image of the jellyfish obtained from the detection experiment, the image of the jellyfish is in the lower left corner, and the corresponding jellyfish posture is shown in the upper right corner. Due to the ability of reflecting sound waves, the acoustic diameter and height of the jellyfish maybe different from the actual jellyfish diameter and height. To some degree, the height of jellyfish is a reflection of the jellyfish posture, when posture varies, the height varies too.
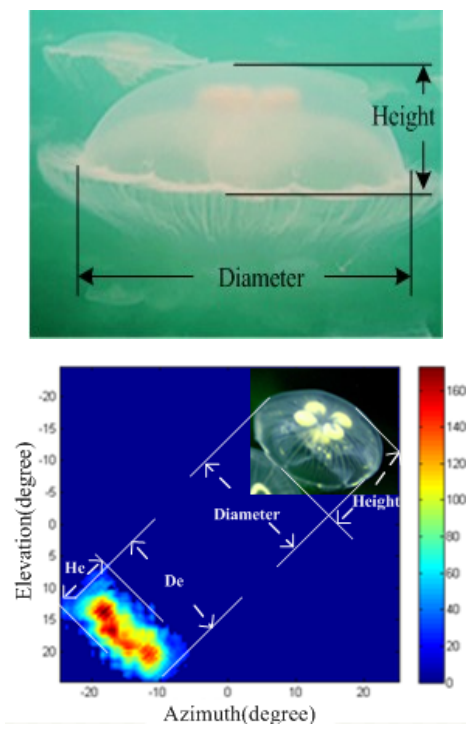

Fig. 2. The diameter and height of jellyfish.

\section{Data analysis and processing}

\subsection{Individual acoustic image of jellyfish}

Fig. 3 is a raw acoustic image of the jellyfishes, which is normalized for the convenience of displaying. The target in Fig. 3 includes a clear image of a jellyfish with a clear image of an individual jellyfish and some obscure jellyfish images. The distance between the nearest jelly fish and the sonar is $0.52 \mathrm{~m}$.

The clear image of the individual jellyfish is abstracted and shown in the Fig. 4 which is also normalized. The echo image of the individual jellyfish consists of two parts: a large background highlight and four smaller but brighter echo highlights.

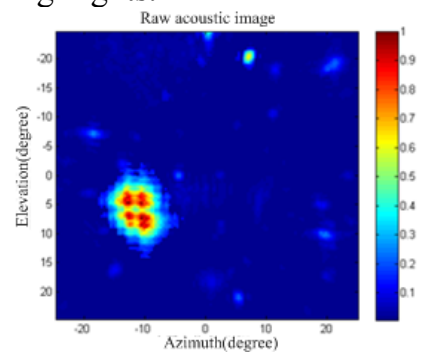

Fig. 3. The jellyfish detection sound image.

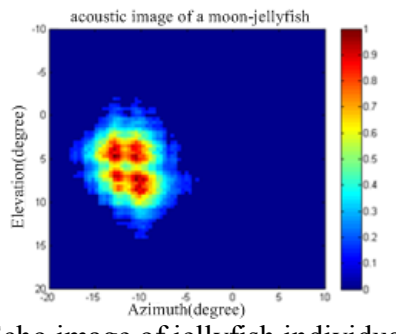

Echo image of jellyfish individual

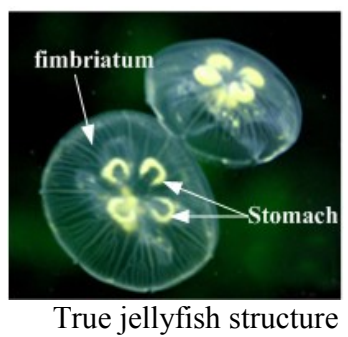

Fig. 4. Echo image of jellyfish individual.

According to biology knowledge, jellyfish body structure is made up of two layers of epithelial cells with middle layer. The middle layer thickness is thicker. The circular stomach rounded by the cavity body wall is a simple capsule, or divided into four stomach capsules by membrane (Fig.4).

Contrasting to the real picture of sea moon jellyfish (Fig.4), it is found that the larger background highlight is due to the echo reflected by the jellyfish umbrella body (the middle layer), and the other four smaller but brighter highlights are due to the four stomach capsules in the moon jellyfish.

\subsection{Estimation of acoustic characteristic parame ters}

Outlines of jellyfish individual echo images are extracted to calculate jellyfish De and He. In Fig.5, there are two processed results of jellyfish individuals, where Fig.5(a) and Fig.5(c) are two jellyfish acoustic images, Fig.5(b) and Fig.5(d) are the contours of acoustic images.

The diameter and height of the two jellyfish individuals are calculated by the image processing method

De1 $=0.15 \mathrm{~m}, \mathrm{He} 1=0.11 \mathrm{~m}$.

$\mathrm{De} 2=0.20 \mathrm{~m}, \mathrm{He} 2=0.10 \mathrm{~m}$.

About fifty moon jellyfish individuals with clear acoustic images are collected to extract acoustic diameter and height parameters. The results are shown in Fig.6. It is shown that the maximum diameter of 
individual jellyfish is $0.20 \mathrm{~m}$, and the minimum value is $0.10 \mathrm{~m}$. The maximum height is $0.13 \mathrm{~m}$ and the minimum height is $0.07 \mathrm{~m}$.

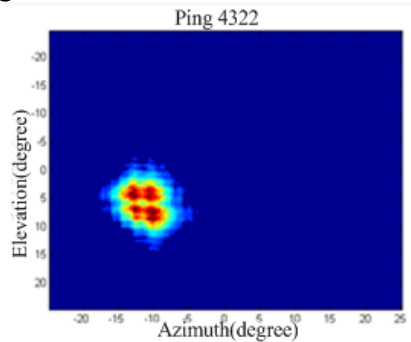

(a)

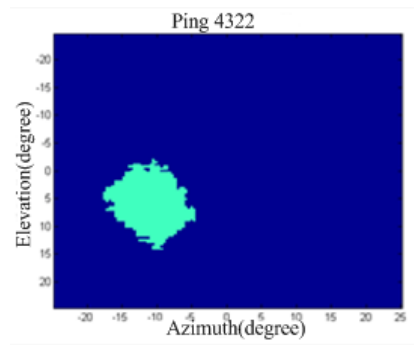

(b)

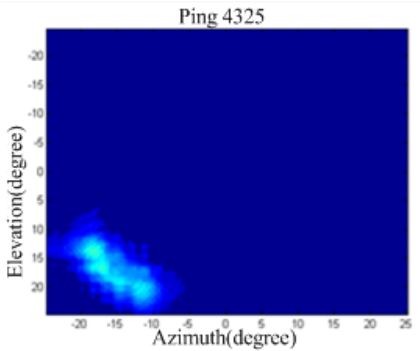

(c)

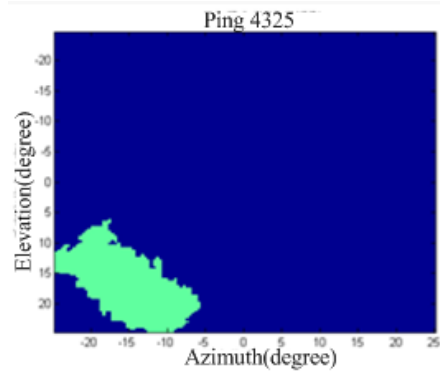

(d)

Fig. 5. The diameter and height of jellyfish individuals.

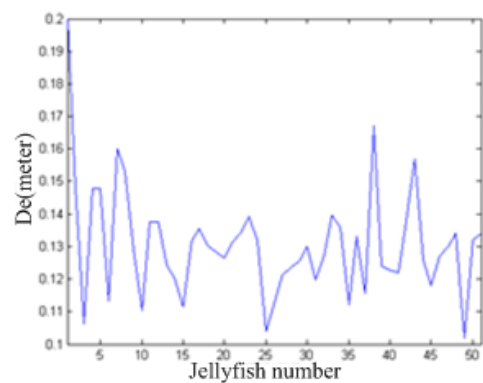

(a)

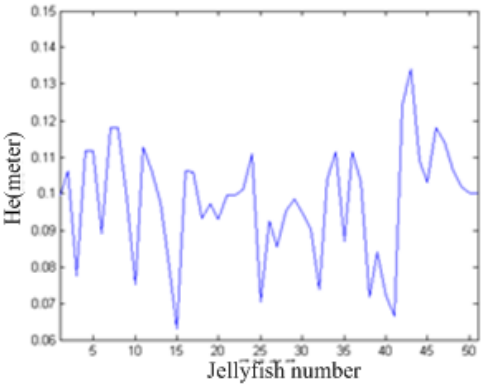

(b)

Fig. 6. The diameter and height of the jellyfish individuals.

The RES of multiple jellyfish individuals are estimated and the results are shown in Fig.7. According to the existing data, the maximum value of the RES is $59.9 \mathrm{~dB}$, and the minimum value is $47.6 \mathrm{~dB}$.

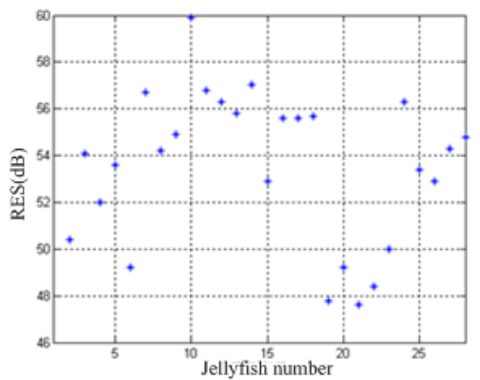

Fig. 7. Relative Echo Strength of moon jellyfishes.

\subsection{The process of jellyfish movement}

In real situation, the jellyfish is in continuous movement. In the paper, the echo characteristics of moving jellyfish are also studied. Fig.1(b) is a schematic diagram of a moving jellyfish, which swims toward the sonar.

It is known that jellyfishes swim in a form of pulsation. The umbrella expands and then rapidly contracts to expel water from the body. By the water-jet approach, the jellyfish can move in the opposite direction. Figure 8 is the acoustic image of the moon jellyfish pulsating toward the sonar. The pulsation of jellyfish is divided into five steps:

Step1. The jellyfish umbrella is completely diastolic and the area of the jellyfish echo image (Fig.8(a)) is the largest, where the RES also reaches the maximum value.

Step2. The jellyfish umbrella begins to contract. The area of the jellyfish echo image (Fig.8(b)), at this time, begins to decrease.

Step3. The jellyfish umbrella shown is total shrinkage. At this time, the area of the jellyfish echo image is the smallest (Fig.8(c)) corresponding to the minimum value of the RES.

Step4. The jellyfish umbrella begins to relax. At this time, the area of the jellyfish echo image (Fig.8(d)) begins to increase.

Step5. The jellyfish umbrella is completely diastolic again. At this time, the area of the jellyfish echo image (Fig.8(e)) is the largest again, corresponding to the maximum value of the RES. 


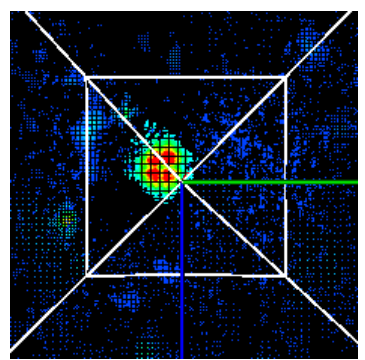

(a)

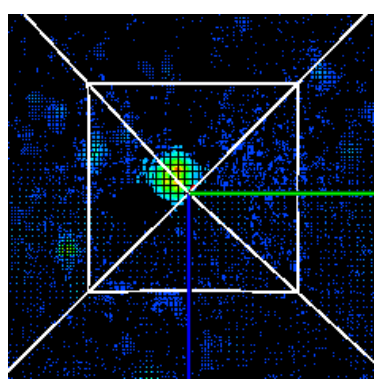

(b)

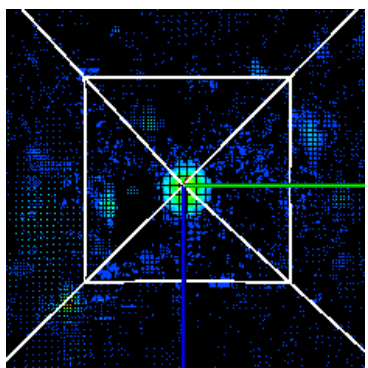

(c)

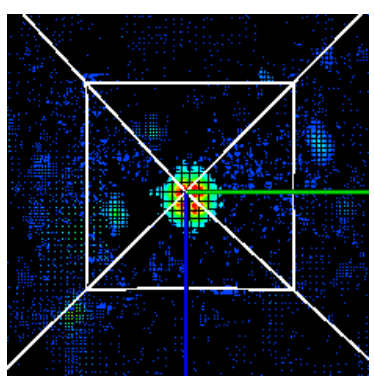

(d)

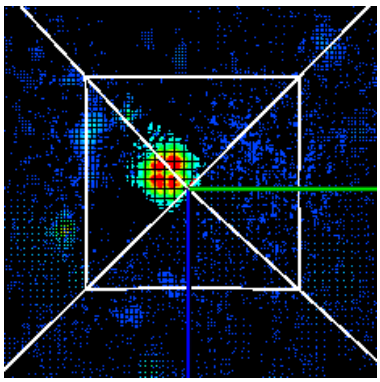

(e)

Fig. 8. The acoustic images of a pulsating moon jellyfish.

The variation of the RES of jellyfish individuals during the pulsation movement is studied. By tracking the pulsating process of two jellyfishes continuously, the RESs during the jellyfish pulsation are estimated and shown in Fig.9.

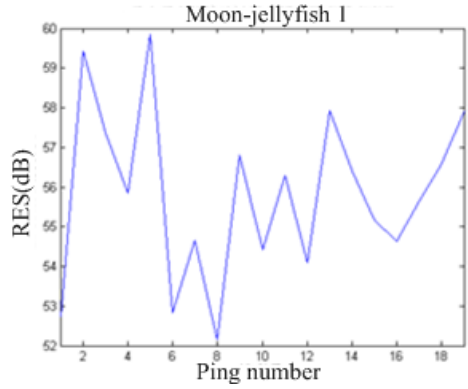

(a)

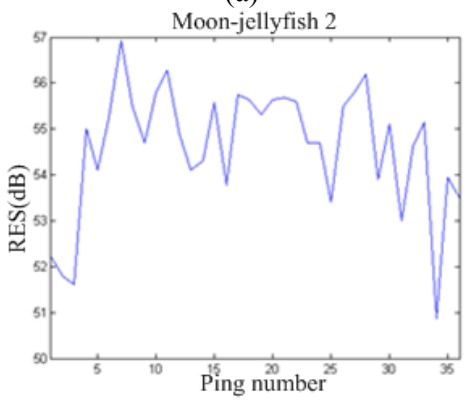

(b)

Fig. 9. RESs during jellyfish pulsation.

During the pulsation of jellyfish 1 , the maximum RES is $59.9 \mathrm{~dB}$, the smallest RES is $52.2 \mathrm{~dB}$, and the variation range is $7.7 \mathrm{~dB}$. During the pulsation of jellyfish 2 , the maximum RES is $56.9 \mathrm{~dB}$, and the smallest RES is $50.9 \mathrm{~dB}$, and the range of changes is 6.0 $\mathrm{dB}$.

\section{Conclusions}

In this paper a method of jellyfish detection based on high resolution multi-beam acoustic image was brought forward. A moon jellyfish detection experiment was conducted on sea. Echo characteristics of individual moon jellyfish were analysed:

(1) The method of jellyfish detection was verified feasible. Clear acoustic images of moon jellyfish individuals were extracted and two kinds of echo highlights due to different parts of jellyfish body were analysed.

(2) Three characteristic parameters were brought up and estimated, which were RES, De and He. These parameters could be used to detect and recognize jellyfish.

(3) RESs of individual moon jellyfish during pulsation were analysed.

\section{References}

1. Lucas, C. H., Gelcich S. \& S. I. Uye, Springer, Dordrecht: 129-150(2014)

2. Song Sun, Hydrobiologia 754:59-74(2015)

3. Jason Rife, IEEE journal of oceanic engineering, 28:595-608 (2003) 